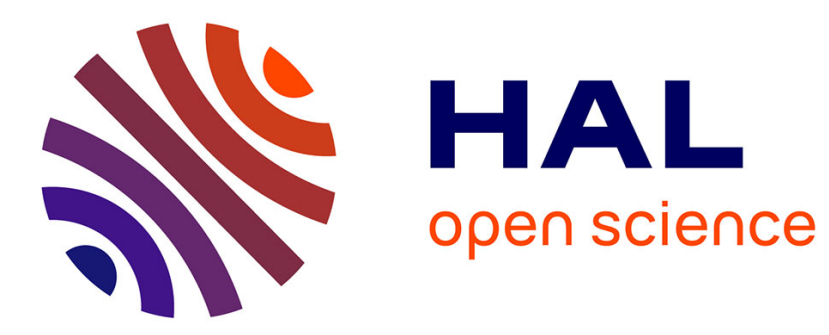

\title{
Concurrent multiscale model of an atomic crystal coupled with elastic continua
}

\author{
P. Deymier, Jerome O. Vasseur
}

\section{To cite this version:}

P. Deymier, Jerome O. Vasseur. Concurrent multiscale model of an atomic crystal coupled with elastic continua. Physical Review B, 2002, 66 (13), 10.1103/PhysRevB.66.134106 . hal-03302147

\section{HAL Id: hal-03302147 \\ https://hal.science/hal-03302147}

Submitted on 27 Jul 2021

HAL is a multi-disciplinary open access archive for the deposit and dissemination of scientific research documents, whether they are published or not. The documents may come from teaching and research institutions in France or abroad, or from public or private research centers.
L'archive ouverte pluridisciplinaire HAL, est destinée au dépôt et à la diffusion de documents scientifiques de niveau recherche, publiés ou non, émanant des établissements d'enseignement et de recherche français ou étrangers, des laboratoires publics ou privés. 


\title{
Concurrent multiscale model of an atomic crystal coupled with elastic continua
}

\author{
P. A. Deymier ${ }^{1, *}$ and J. O. Vasseur ${ }^{2}$ \\ ${ }^{1}$ Department of Materials Science and Engineering, The University of Arizona, Tucson, Arizona 85721 \\ ${ }^{2}$ Laboratoire de Dynamique et Structures des Matériaux Moléculaires, UPRESA CNRS 8024, UFR de Physique, Université de Lille I, \\ 59655 Villeneuve d'Ascq Cédex, France \\ (Received 28 May 2002; revised manuscript received 23 August 2002; published 30 October 2002)
}

\begin{abstract}
A methodology based on a Green's function formalism, which enables the coupling between atomic and continuous systems, is employed to evaluate the dynamical properties of concurrent elastic multiscale models. Boundary conditions satisfying the continuity of displacement and stress across interfaces between a harmonic crystal and continua are insufficient to establish seamless coupling at high frequency. The elastic mismatch in coupled discrete/continuous models is inherently linked to the difference in dispersion of the constitutive media.
\end{abstract}

DOI: 10.1103/PhysRevB.66.134106

PACS number(s): 68.35.Ja, 62.30.+d, 63.20.-e, 68.65. $-\mathrm{k}$

\section{INTRODUCTION}

Multiscaling has recently received much attention in several branches of physical science. Existing multiscale simulation methodologies can be characterized as serial or concurrent. Within serial methods, a set of calculations at a fundamental level (small length scale) is used to evaluate parameters as input for a more phenomenological model that describes a system at longer length scales. For example the quasicontinuum method is a zero temperature technique with a formulation based on standard continuum mechanics [e.g., the finite element (FE) method] with the additional feature that the constitutive equations are drawn from calculations at the atomic scale. ${ }^{1-4}$ In contrast, concurrent methods build around the idea of describing the physics of different regions of a material with different models and linking them via a set of boundary conditions. The archetype of concurrent methods divides the space into atomistic regions coupled with a continuum modeled via the FE method. ${ }^{5,6}$ Coarse graining has been proposed as a mean to couple seamlessly a molecular dynamics (MD) region to a FE mesh. ${ }^{7}$ Coarse-grained MD produces equations of motion for a mean-displacement field at the nodes of a coarse-grained mesh partitioning the atomistic system. Other algorithms that allow the coupling between atomistic and continuum regions have been proposed. ${ }^{8-11}$ Broughton et al. ${ }^{8}$ presented an algorithm involving hand shaking between FE and MD methods. This algorithm was able to dynamically track a crack propagating through silicon. The handshaking between the MD and FE regions was achieved by drawing an imaginary surface between them. Within the range of the MD interatomic potential from this surface, FE mesh points were located at equilibrium atomic sites. Any FE element that crosses the interface contributes half its weight to a conservative Hamiltonian. Similarly any MD interaction that crosses the interface also contributes half its weight to this Hamiltonian. Kohlhoff et al. ${ }^{9}$ introduced a similar transition region between the atomic and continuous regions. They also scaled down the finite element size to the atomic scale in this transition region. Unlike Broughton et al.'s work, the interface was of finite size and not sharp. Abraham et al. ${ }^{10}$ combined the above two techniques by constructing an explicit Hamil- tonian for the atoms and the FE nodes in the transition region by weighing their contributions with respect to their distance away from the middle of the interface. Ogata et al. ${ }^{11}$ used a similar algorithm to study chemical reactions and their interplay with mechanical phenomena in materials, such as in the oxidation of the $\mathrm{Si}(111)$ surface.

In the case of dynamical simulations, interfaces between atomistic systems and the coarser FE mesh will suffer unwanted reflections as the second medium cannot support short-wavelength vibrational modes. This problem could be overcome by coupling an atomistic system to a true continuum and not one of its discretized forms. Indeed, a continuum exhibits a linear dispersion relation for all frequencies. Here we consider an approach based on Green's functions wherein a hybrid system is constructed by interfacing a harmonic cubic crystal and an elastic continuum through appropriate boundary conditions. The boundary conditions satisfy the continuity of elastic displacements and the continuity of stresses. The method naturally avoids undesirable effects due to the discretization of the continuum. It retains, however, the inherent difference in vibrational behavior of the nonlinear dispersive atomic system and of the linear dispersive continuum. The investigation of the effect of this difference on the propagation of elastic waves in discrete/continuum composite media constitutes the core of the present study. We consider two hybrid systems, namely, (A) a semi-infinite continuum/semi-infinite crystal and, (B) a finite crystal slab sandwiched between two semi-infinite continua. The first system provides a window on the properties of a single interface between continuum and atomic media. The necessity for multiscale modeling and simulation of hybrid continuum and atomic systems finds its roots in a need for minimization of computational effort. To that effect, the atomic scale region typically takes on finite dimensions and is embedded into a continuum with larger dimensions. The continuum/discrete/continuum system (B) provides the basis for the quantification of undesirable effect in such hybrid systems.

In Sec. II, we present in details the Green's functionbased methodology enabling the coupling between elastic continua and harmonic crystals. Plane waves propagating through the hybrid systems are used to probe and quantify 


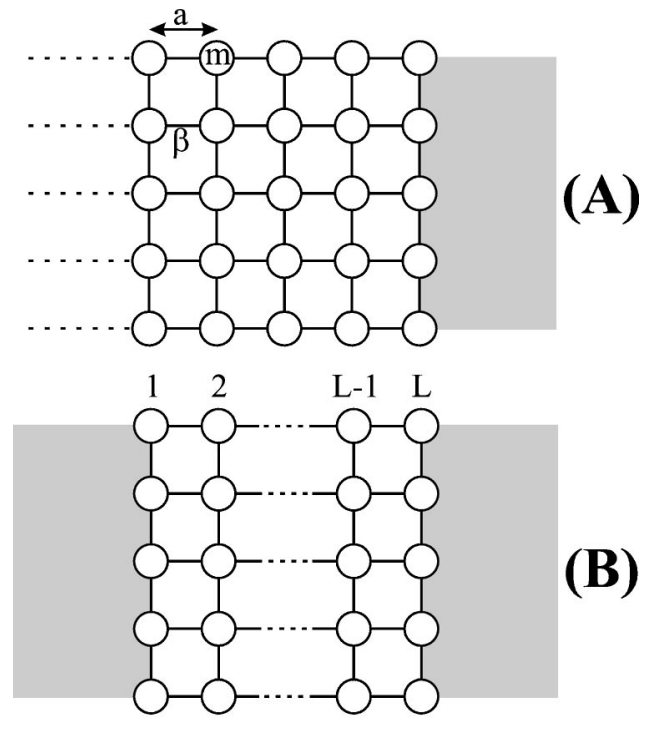

FIG. 1. Projections of (A) the semi-infinite harmonic atomic crystal/semi-infinite continuum and (B) the semi-infinite continuum/crystal slab/semi-infinite continuum sandwich. The gray areas represent the continua. See the text for details.

the coupling between the continuum and discrete media. In Sec. III, we report the transmission coefficient of the hybrid systems (A) and (B) as a function of the angular frequency. Conclusions concerning the applicability and limitations of concurrent discrete/continuous multiscale models are drawn in Sec. IV.

\section{MODELS AND METHODOLOGY}

We treat two coupled systems, namely, (A) a semi-infinite continuum/semi-infinite crystal, and (B) a finite crystal slab sandwiched between two semi-infinite continua. In both cases, the interfaces are taken to be parallel to a (001) plane (see Fig. 1). We exploit the periodicity of the continuum and of the crystal in the direction parallel to the interface to decompose the elastic and the vibrational Green's functions (and displacement) of the semi-infinite media and slab in Fourier series in the coordinates $\vec{k}_{/ /}$, parallel to the interfaces. For the sake of simplicity, we reduce the problem to wave propagation along the direction perpendicular to the interfaces by setting $\vec{k}_{/ /}=\overrightarrow{0}$. We also limit this study to transverse modes of vibration. The equation of motion in the continuum simplifies to the one-dimensional elastic wave equation. Assuming that the excitations execute harmonic motion with pulsation $\omega(\omega=2 \pi \nu$ where $\nu$ is the frequency) and a time dependence $\exp (-i \omega t)$, the continuum equation of motion becomes

$$
\rho \omega^{2} u+C_{44} \frac{\partial^{2} u}{\partial x^{2}}=0
$$

where $u$ is the displacement and $x$ the direction [001]. The elastic continuum has the density, $\rho$. The Green's function of the bulk continuous elastic medium, $G^{c}$, is given by the equation

$$
\rho \omega^{2} G^{c}\left(x, x^{\prime}\right)+C_{44} \frac{\partial^{2} G^{c}\left(x, x^{\prime}\right)}{\partial x^{2}}=\delta\left(x-x^{\prime}\right),
$$

which has, for a solution, ${ }^{12}$

$$
G^{c}\left(x, x^{\prime}\right)=-\frac{1}{2 \rho \alpha_{t} C_{t}^{2}} \exp \left(-\alpha_{t}\left|x-x^{\prime}\right|\right) .
$$

In Eq. (2), $\delta$ is the usual delta function. The elastic constant $C_{44}$ is related to the density and transverse speed of sound through $C_{t}=\sqrt{C_{44} / \rho} . \alpha_{t}$ is defined by the relation $\alpha_{t}^{2}=k_{/ /}^{2}$ $-\left(\omega / C_{t}\right)^{2}$ which, at $k_{/ /}=0$ yields $\alpha_{t}=-i\left(\omega / C_{t}\right)$.

The atomic system will be taken to be a simple-cubic crystal with lattice parameter $a$. The atoms have mass $m$. The lattice vibrations of the crystal are described with the Montroll-Potts model. ${ }^{13}$ The interactions between nearest neighbor atoms are harmonic with the isotropic force constant coefficients $\beta$. The equation of motion for transverse waves in the discrete system for $\vec{k}_{/ /}=\overrightarrow{0}$ is that of a onedimensional monoatomic harmonic crystal, namely,

$$
m \omega^{2} u_{n}-\beta\left(u_{n+1}-2 u_{n}+u_{n-1}\right)=0 .
$$

The index $n$ refers to an atomic site along the [001] direction.

From Eq. (4) and using $\rho=m / a,{ }^{3}$ it is straightforward to obtain a relationship dimensionally equivalent to Eq. (2) that defines the Green's function of the discrete system, $G^{d}$,

$$
\beta G^{d}\left(n+1, n^{\prime}\right)-\gamma G^{d}\left(n, n^{\prime}\right)+\beta G^{d}\left(n-1, n^{\prime}\right)=a^{2} \delta_{n, n^{\prime}},
$$

where $\delta_{n, n}$ is the Kroenecker symbol and $\gamma=2 \beta-m \omega^{2}$. In deriving Eq. (5), it is worth noting that the Kroenecker symbol is a dimensionless quantity while the delta function in Eq. (2) has dimension of the inverse of a length. The solution to Eq. (5) is well known, ${ }^{14}$ and is given as

$$
G^{d}\left(n, n^{\prime}\right)=\frac{a^{2}}{\beta} \frac{t^{\left|n-n^{\prime}\right|+1}}{t^{2}-1}
$$

with

$$
t= \begin{cases}\xi-\sqrt{\left(\xi^{2}-1\right)} & \text { if } \xi>1 \\ \xi+\sqrt{\left(\xi^{2}-1\right)} & \text { if } \xi<-1 \\ \xi+i \sqrt{\left(1-\xi^{2}\right)} & \text { if }-1<\xi<+1\end{cases}
$$

and

$$
\xi=\frac{\gamma}{2 \beta}
$$

The transverse speed of sound, $C_{t}$, in the continuum medium is related to the long-wavelength limit of the crystal wave velocity according to $C_{t}=a \sqrt{\beta / m}$. The elastic Green's function for the semi-infinite continuum with a stress free surface located at $x=0, g_{s}^{c}$ was also reported in Ref. 12: 


$$
\begin{aligned}
g_{s}^{c}\left(x, x^{\prime}\right)= & -\frac{1}{2 \rho \alpha_{t} C_{t}^{2}}\left\{\exp \left(-\alpha_{t}\left|x-x^{\prime}\right|\right)\right. \\
& \left.+\exp \left[-\alpha_{t}\left(x+x^{\prime}\right)\right]\right\} \text { if } x, x^{\prime}>0 .
\end{aligned}
$$

The Green's functions of a semi-infinite discrete crystal, $g_{s}{ }^{d_{1}}$ and of a discrete crystal slab composed of $L(001)$ atomic planes with two parallel ideal free surfaces, $g_{s}^{d_{2}}$, are calculated in Ref. 14:

$$
\begin{aligned}
g_{s} d_{1}\left(n, n^{\prime}\right)= & \frac{a^{2}}{\beta} \frac{t^{\left|n-n^{\prime}\right|+1}+t^{2-\left(n+n^{\prime}\right)}}{t^{2}-1} \text { if } n, n^{\prime} \leqslant 0, \\
g_{s}^{d_{2}}\left(n, n^{\prime}\right)= & \frac{a^{2}}{\beta}\left\{\frac{t^{\left|n-n^{\prime}\right|+1}+t^{n+n^{\prime}}}{t^{2}-1}+\frac{t^{2 L+1}}{\left(t^{2}-1\right)\left(1-t^{2 L}\right)}\right. \\
& \left.\times\left(t^{n-n^{\prime}}+t^{n^{\prime}-n}+t^{1-n-n^{\prime}}+t^{n+n^{\prime}-1}\right)\right\} \\
& \text { if } 1 \leqslant n, n^{\prime} \leqslant L .
\end{aligned}
$$

These dynamical response functions depend on the angular frequency $\omega$. We use a general theory of response functions for partly discrete and partly continuous inhomogeneous systems, ${ }^{15}$ originally applied to a tight binding/nearly free electron composite, to the calculation of the Green's function of the coupled systems (A) and (B). This theory builds upon the interface response theory of discrete composite systems ${ }^{16}$ and that of continuous composite materials. ${ }^{17}$ The elastic displacement field $u(D)$ everywhere within the space $D$ of the composite system is given by

$$
\begin{aligned}
u(D)= & U(D)-U(M) G^{-1}(M M) G(M D) \\
& +U(M) G^{-1}(M M) g(M M) G^{-1}(M M) G(M D)
\end{aligned}
$$

where $M$ refers to the space of the interfaces. $G$ stands for the bulk Green's functions of the constitutive blocks of the composite. $U$ is a bulk reference displacement field also defined in the constitutive blocks. $g(M M)$ is the Green's function of the composite medium limited to its space of interfaces. The elements of the inverse of $g(M M)$ belonging to the interfaces between continuous and discrete systems are obtained as the sum of elements of the inverses of the $g_{s}^{\prime} s$ of the abutting constitutive blocks, such that

$$
\begin{gathered}
g^{-1}\left(\mathbf{r} \in M_{i j}, \mathbf{r}^{\prime} \in M_{k l}\right)=0 \quad \text { if } \quad M_{k l} \notin M_{i}, \quad(12 \mathrm{a}) \\
g^{-1}\left(\mathbf{r} \in M_{i j}, \mathbf{r}^{\prime} \in M_{i l}\right)=g_{s}^{-1}\left(\mathbf{r} \in M_{i j}, \mathbf{r}^{\prime} \in M_{i l}\right) \quad \text { if } l \neq j,
\end{gathered}
$$

$$
g^{-1}\left(\mathbf{r} \in M_{i j}, \mathbf{r}^{\prime} \in M_{i j}\right)=\sum_{k} g_{s}^{-1}\left(\mathbf{r} \in M_{k l}, \mathbf{r}^{\prime} \in M_{k l}\right)
$$$$
\text { if } M_{k l} \equiv M_{i j}
$$

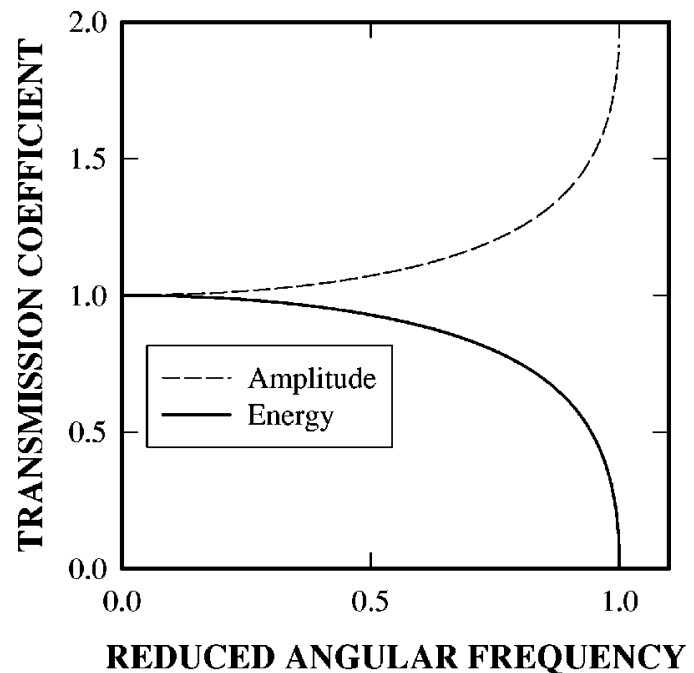

FIG. 2. Transmission coefficient in energy (solid line) and amplitude (dashed line) vs the reduced angular frequency of an incident plane wave launched from a semi-infinite elastic continuum and transmitted into the coupled semi-infinite simple cubic harmonic crystal.

with $M_{i j}$ standing for the interface between the constitutive blocks $i$ and $j . M_{i}$ represents the space of the interfaces of block $i$. This superposition of the inverses of surface Green's functions imposes the appropriate boundary conditions of continuity of displacements and of continuity of stresses at the interfaces. The application of this method to system (A) is detailed in the Appendix.

\section{RESULTS}

First we investigate the single interface between a harmonic crystal and the elastic continuum [system (A)] by calculating, according to Eq. (11) (see the Appendix), the transmitted displacement field $u$ in the discrete system when a plane wave reference displacement field $U$ is launched in the continuous medium. In Fig. 2, we report the amplitude transmission coefficient $\tau=|u / U|^{2}$ and the energy transmission coefficient, defined as $T=\left(Z_{d} / Z_{c}\right) \tau$, as functions of reduced angular frequency, $\omega^{*}=\omega / \omega_{\max }$ with $\omega_{\max }=2 \sqrt{\beta / m}$. Here $Z_{d}=\rho V_{g}$ and $Z_{c}=\rho C_{t}$ are the impedances of the continuous and discrete media, respectively. $V_{g}$ is the group velocity of the atomic system. It is frequency dependent and determined from the dispersion relation of the crystal. At low frequencies and long wavelengths, there is no impedance mismatch between the continuum and the crystal and both transmission coefficients approach the ideal value of 1 . The transmission of energy is impeded as the frequency approaches the upper limit of the crystal vibrational band. Beyond $\omega_{\max }$, all waves are reflected at the interface. The monotonic decrease in the energy transmission coefficient from 1 to 0 as the frequency increases results in part from the fact that the transverse speed of sound of the continuum remains constant while the group velocity of the crystal diminishes from the continuum 

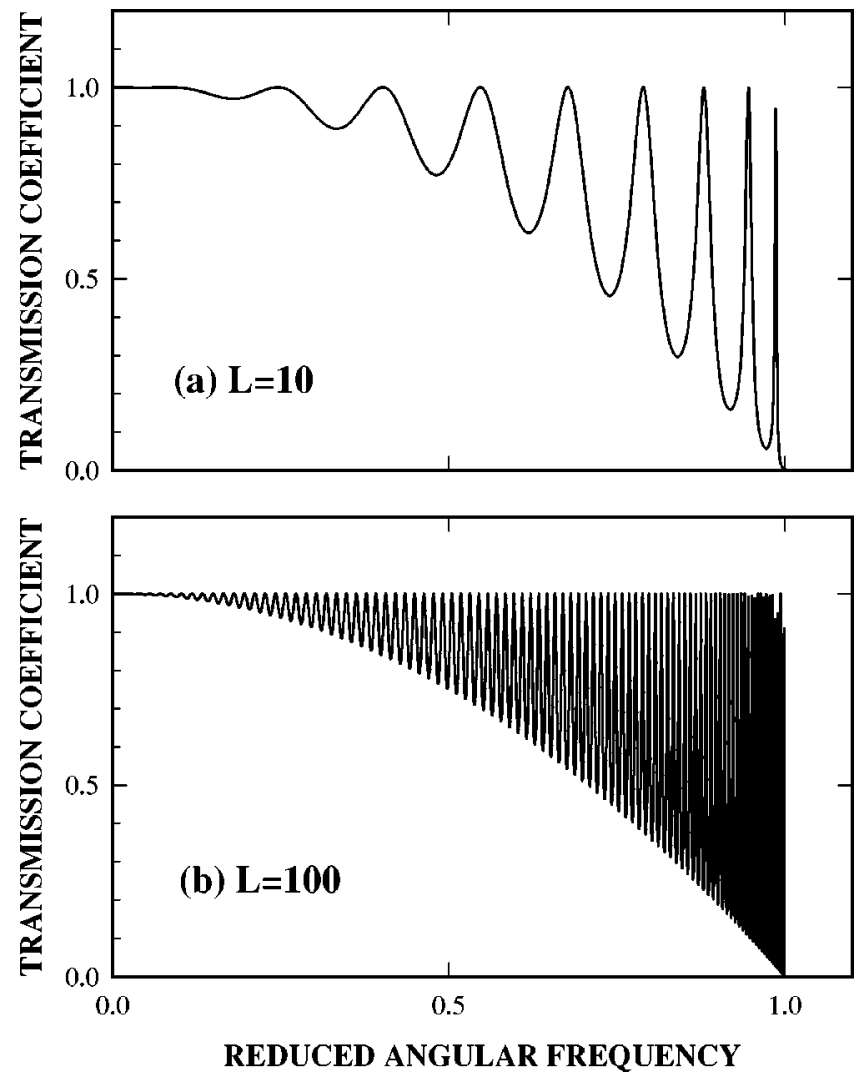

FIG. 3. Transmission coefficient vs the reduced angular frequency for the continuum/crystal slab/continuum sandwich. The slab crystal has (a) 10 and (b) 100 (001) planes.

limit to zero at the highest frequency. The amplitude transmission coefficient does not have to be bounded by 1 as it does not need to satisfy a conservation law as is the case for energy. At high frequency the continuum appears relatively stiffer than the crystal yielding an increase in amplitude transmission coefficient.

In the continuum/discrete/continuum system (B), the space $M$ includes two interfaces parallel to the (001) plane. The slab is finite in the direction [001]. The thickness of the crystal slab is variable and is defined by $L$, the number of (001) atomic planes perpendicular to that direction. We launch a plane wave reference displacement in one of the semi-infinite continua, and calculate the transmitted amplitude in the other. The transmission coefficients in amplitude and in energy are identical in this instance since the emitting and receiving media have the same impedance. The variation of the transmission coefficient with reduced angular frequency for two slab thicknesses is reported in Fig. 3. Similarly to system (A) and owing to the boundary conditions, a perfect match between the continua and the finite crystal is achieved at long wavelengths. The transmission vanishes at the top of the crystal band. Contrary to the single interface, however, a $100 \%$ transmission occurs at selected frequencies throughout the vibrational band of the crystal. These frequencies correspond to resonances in the continuum band associated with the discrete vibrational modes of the finite slab. The number of resonant modes is directly dependent on the number of (001) planes constituting the slab. While the

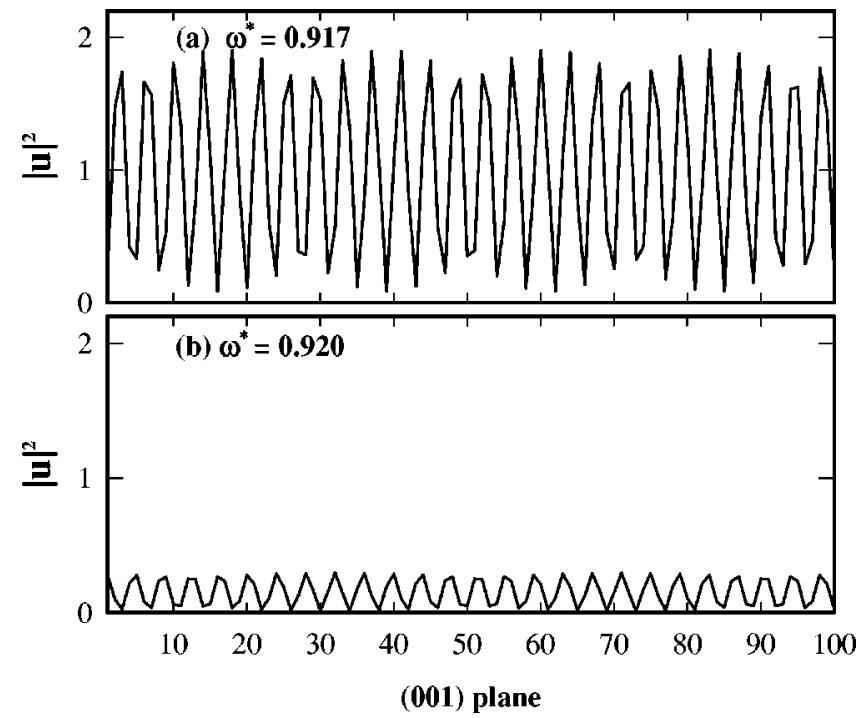

FIG. 4. Spatial variation of the square of the modulus of the amplitude of the displacement field inside the crystal slab coupled to two semi-infinite continua. The slab has $L=100$ (001) planes, and the frequencies (a) and (b) correspond to a maximum and a minimum in transmission, respectively.

number of transmitting frequencies increases with the slab thickness, the total energy transmitted from one continuum to the other remains constant at a value of approximately $78.5 \%$. We have defined the total energy transmitted as the integral of the transmission coefficient over the complete crystal band. We also calculate the displacement field, $u$, inside the finite crystal slab with Eq. (11) in the case of a plane wave reference state $U$. If the crystal slab were part of an infinite atomic crystal the reference plane wave state would propagate freely and the square of its amplitude would remain uniform and equal to 1 throughout the entire slab. The displacement field inside the coupled slab, however, loses its plane wave character. The square of the modulus of the displacement field is illustrated in Fig. 4 at two high frequencies corresponding to a maximum and a subsequent minimum in transmission. At maximum transmission the displacement amplitude oscillates about the plane wave uniform value of 1. A minimum in transmission results from a significant reduction in the displacement amplitude. In both cases the amplitude modulus varies spatially. These variations have a standing wave character and at high frequency exhibit modulations at short and long spatial scales. These variations are well seen in the limit of the complex amplitude of the displacement field inside the slab for $\omega^{*} \rightarrow 1$. This limit takes the form

$$
u\left(n, \omega^{*}\right) \approx(-1)^{n-1} \frac{(1-L)-i L}{(1-L)^{2}+L^{2}} 2(L-n) \sqrt{2\left(1-\omega^{*}\right)},
$$

where $n$ stands for the atomic plane and $1 \leqslant n \leqslant L$. In sharp contrast the reference plane wave state has the limit

$$
U_{p w}\left(n, \omega^{*}\right) \approx(-1)^{n-1}\left[-1+i 2 n \sqrt{2\left(1-\omega^{*}\right)}\right]
$$

whose real part remains spatially uniform and non-zero at the top of the crystal band. 


\section{CONCLUSION}

In conclusion, we have presented a methodology based on Green's functions for coupling atomic and continuous media. This approach overcomes the undesirable reflections due to the discretization of the continuum commonly encountered in hybrid atomic/FE multiscale methods. The boundary conditions of continuity of displacements and stresses at the interface between the elastic continuum and the atomic harmonic crystal yield seamless coupling only at long wavelengths. The transmission of the elastic wave energy is significantly impeded at frequencies approaching the top of the vibrational band of the atomic system due to the mismatch in the dispersion relations of the continuous and discrete media. A finite atomic crystal embedded in a continuum supports resonant vibrational modes leading to maxima and minima in the transmission spectrum of the coupled system. The size of the finite crystal affects the number of resonances (i.e., frequencies at which transmission occurs) but does not impact the total energy that can be transmitted. Short and long scale modulations of the displacement field inside the coupled finite crystal may constitute a strong limitation in the applicability of concurrent discrete/continuous multiscale models in representing correctly the physics of large atomic systems.

\section{ACKNOWLEDGMENTS}

One of us (J.O.V.) would like to acknowledge the hospitality of the Department of Materials Science and Engineering at the University of Arizona where this work was done. This work was supported in part by NSF Grant No. 9980015. We thank B. Djafari-Rouhani and J. Simmons for fruitful discussions.

\section{APPENDIX}

The space of the interface, $M$, between the discrete and the continuous media in system (A) is located at $x=0$ ( $n$ $=0)$. Using Eqs. (8) and (9), we calculate the surface Green's functions of the two media in $M$ :

*Corresponding author. Email address: deymier@u.arizona.edu

${ }^{1}$ E. B. Tadmor, M. Ortiz, and R. Phillips, Philos. Mag. A 73, 1529 (1996).

${ }^{2}$ E. B. Tadmor, R. Phillips, and M. Ortiz, Langmuir 12, 4529 (1996).

${ }^{3}$ V. B. Shenoy, R. Miller, E. B. Tadmor, R. Phillips, and M. Ortiz, Phys. Rev. Lett. 80, 742 (1998).

${ }^{4}$ G. S. Smith, E. B. Tadmor, and E. Kaxiras, Phys. Rev. Lett. 84, 1260 (2000).

${ }^{5}$ M. Mullins and M. A. Dokainish, Philos. Mag. A 46, 771 (1982).

${ }^{6}$ H. Kitagawa, A. Nakatami, and Y. Sibutani, Mater. Sci. Eng., A 176, 263 (1994).

${ }^{7}$ R. E. Rudd and J. Q. Broughton, Phys. Rev. B 58, R5893 (1998).

${ }^{8}$ J. Q. Broughton, F. F. Abraham, N. Bernstein, and E. Kaxiras, Phys. Rev. B 60, 2391 (1999).

$$
\begin{aligned}
& g_{s}^{c}\left(x=0, x^{\prime}=0\right)=-\frac{1}{\rho \alpha_{t} C_{t}^{2}}, \\
& g_{s}{ }^{d_{1}}\left(n=0, n^{\prime}=0\right)=\frac{a^{2}}{\beta} \frac{t}{t-1} .
\end{aligned}
$$

The inverse of the Green's function of system (A) in $M$ is therefore obtained by inverting expressions (A1a) and (A1b) and inserting them into Eq. (12c). That is,

$$
g^{-1}(0,0)=\frac{\beta}{a^{2}} \frac{t-1}{t}-\rho \alpha_{t} C_{t}^{2} .
$$

We calculate the displacement field at atomic site, $n$, in the discrete medium from a reference unit amplitude plane wave in the continuous medium. For system (A) with a single interface, Eq. (11) takes the simple form

$$
\begin{aligned}
u(n)= & U(n)-U(x=0) G^{-1}\left(x=0, n^{\prime}=0\right) G\left(n^{\prime}=0, n\right) \\
& +U(x=0) G^{-1}\left(x=0, x^{\prime}=0\right) g(0,0) \\
& \times G^{-1}\left(n^{\prime}=0, n^{\prime \prime}=0\right) G\left(n^{\prime \prime}=0, n\right) .
\end{aligned}
$$

Since $U$ is only defined in the continuous medium, the first term of equation (A3) vanishes. The inverse of the bulk Green's function $G^{-1}$ in the second term of Eq. (A3) is not defined for $x=0$ and $n^{\prime}=0$ since these locations correspond to two different media. The second term also vanishes. For system (A), only the third term of Eq. (A3) survives and becomes:

$$
\begin{aligned}
u(n)= & U(x=0)\left[G^{c}\left(x=0, x^{\prime}=0\right)\right]^{-1} g(0,0) \\
& \times\left[G^{d}\left(n^{\prime}=0, n^{\prime \prime}=0\right)\right]^{-1} G^{d}\left(n^{\prime \prime}=0, n\right) .
\end{aligned}
$$

Using $U(0)=1$, Eq. (3) for $G^{c}$, Eq. (6) for $G^{d}$, and Eq. (A2) for $g$, one obtains

$$
u(n)=-2 \rho \alpha_{t} C_{t}^{2} \frac{1}{\frac{\beta}{a^{2}} \frac{t-1}{t}-\rho \alpha_{t} C_{t}^{2}} t^{-n} .
$$

The transmitted displacement field in system (B) can be obtained in a similar way.

${ }^{9}$ S. Kohlhoff, P. Gumbsch, and H. F. Fischmeister, Philos. Mag. A 64, 851 (1991).

${ }^{10}$ F. F. Abraham, J. Q. Broughton, N. Bernstein, and E. Kaxiras, Comput. Phys. 12, 538 (1998).

${ }^{11}$ S. Ogata, E. Lidorikis, F. Shimojo, A. Nakano, P. Vashista, and R. K. Kalia, Comput. Phys. Commun. 138, 143 (2001).

${ }^{12}$ A. A. Maradudin and D. L. Mills, Ann. Phys. (N.Y.) 100, 262 (1976).

${ }^{13}$ E. W. Montroll and R. B. Potts, Phys. Rev. 102, 72 (1956).

${ }^{14}$ A. Akjouj, B. Sylla, and L. Dobrzynski, Ann. Phys. (Paris) 18, 363 (1993).

${ }^{15}$ A. Akjouj, L. Dobrzynski, and C. Oleksy, Surf. Sci. 213, 630 (1989).

${ }^{16}$ L. Dobrzynski, Surf. Sci. Rep. 6, 119 (1986).

${ }^{17}$ L. Dobrzynski, Surf. Sci. 180, 489 (1987). 